\title{
THE INDEX OF A BRAUER CLASS \\ ON A BRAUER-SEVERI VARIETY
}

\author{
AIDAN SCHOFIELD AND MICHEL VAN DEN BERGH
}

\begin{abstract}
Let $D$ and $E$ be central division algebras over $k$; let $K$ be the generic splitting field of $E$; we show that the index of $D \otimes_{k} K$ is the minimum of the indices of $D \otimes E^{\otimes i}$ as $i$ varies. We use this to calculate the index of $D$ under related central extensions and to construct division algebras with special properties.
\end{abstract}

\section{INTRODUCTION}

A question that seems not to have been discussed is the following. Let $D$ and $E$ be central division algebras over a field $k$; let $K$ be the function field of the Brauer-Severi variety, $\mathrm{Br}-S(E)$, what is the index of $D \otimes_{k} K$ ? It is this question that we shall answer. We show that it is the minimum of the indices of $D \otimes E^{\otimes i}$ as $i$ varies.

Section 1 contains two proofs of this result. The first proof gives an upper bound for the index of $D \otimes_{k} K$ where $K$ is the function field of any variety over $k$, and this bound yields the result we want. The second proof uses the $K$-theory of the Brauer-Severi variety, $\mathrm{Br}-S(E)$. Quillen [6] shows that $K_{*}(\mathrm{Br}-S(E)) \simeq \bigoplus_{i=0}^{n-1} K_{*}\left(E^{\otimes i}\right)$ where $n$ is the index of the division algebra $E$. We make sense of $K_{*}\left(D \otimes_{k} \mathrm{Br}-S(E)\right)$ which we show is isomorphic to $\bigoplus_{i=0}^{n-1} K_{*}\left(D \otimes_{k} E^{\otimes i}\right)$, and this result yields the main theorem very quickly.

Section 2 is devoted to consequences of this result. Thus, if $D$ is a division algebra of index $p^{t}$ for some prime $p$, and $K$ is the function field of the Brauer-Severi variety of $D^{p^{s}}, s>0$, then $D \otimes_{k} K$ is still a division algebra. Here, we should note that $D^{\otimes m}$ denotes the central simple algebra

$$
D \otimes \underset{(m \text { times })}{D \otimes \cdots} \otimes D
$$

and $D^{m}$ denotes the division algebra Morita equivalent to $D^{\otimes m}$. We will never use the notation $D^{m}$ in this sense when $m=0$, so that no confusion with the opposite algebra can arise.

Refinements of the result above show simply that the generic division algebras of index $p^{t}$ and exponent $p^{s}$ for $s>1$ are indecomposable for all primes $p$. This application was pointed out by David Saltman.

Received by the editors October 5, 1989 and, in revised form, July 9, 1990.

1980 Mathematics Subject Classification (1985 Revision). Primary 12E15, 16A39. 
We discuss the behavior of the index $D$ under other generic central extensions; thus if the index of $D$ is $n$, it is possible to find a field $K(m) \supset k$ such that $D \otimes_{k} K(m)$ has index $m$ for any given divisor $m$ of $n$.

Again there is a field $K \supset k$ such that $D \otimes_{k} K$ is a cyclic division algebra (see the discussion after 2.6).

Our last example is a pair of division algebras $D$ and $E$ of index $p$ for any odd prime such that $D \otimes E^{0}$ has index $p^{2}$ but $D \otimes E^{i}$ has index $p$ for all $i \not \equiv-1,0 \bmod p$. Thus $D$ and $E$ have no subfield in common. This complements a result of Tignol and Wadsworth [8].

\section{The MAIN RESULT}

We begin by recalling some relevant geometric facts and defining the numerical invariant we require.

Let $\bar{k}$ be the separable closure of $k$. If $X$ is a scheme defined over $k$ then we will denote $X \times_{k} \bar{k}$ by $\bar{X}$. If $\mu: X \rightarrow Y$ is a map between $k$-schemes and $\mathscr{F}$ is a quasicoherent sheaf on $X$ then $\bar{\mu}$ and $\bar{F}$ are defined similarly.

Let $Y$ be an algebraic variety over $k$, not necessarily projective and let $G$ be the Galois group of $\bar{k} / k$. The Hochschild-Serre spectral sequence for etale cohomology [5, Theorem III 2.20, Remark 2.21] gives an exact sequence:

$$
0 \rightarrow \operatorname{Pic}(Y) \rightarrow \operatorname{Pic}^{G}(\bar{Y}) \stackrel{\phi(Y)}{\longrightarrow} \operatorname{Br}(k) \rightarrow \operatorname{Br}(\bar{Y} / Y) \rightarrow H^{1}(G, \operatorname{Pic}(\bar{Y}))
$$

which is functorial in $Y . \operatorname{Pic}^{G}(\bar{Y})$ is the group of $G$-invariant line bundles on $\bar{Y}$, and $\operatorname{Br}(\bar{Y} / Y)$ is that subgroup of $\operatorname{Br}(Y)$ split by $\bar{Y}$.

Here, and in the sequel, a $G$-invariant line- or vector bundle will be one that is invariant up to isomorphism.

If $\mathscr{L}$ is a representative of a $G$-invariant class in $\operatorname{Pic}(\bar{Y})$, and $\Gamma(\mathscr{L})$ is of dimension $n$, then as in the proof of [4, Theorem 3], $\phi(Y)(\mathscr{L})=-[S]$ where $S$ is a central simple $k$-algebra of dimension $n^{2}$ over $k$. (Note that in loc. cit. it is assumed that $Y$ is smooth projective, but this is not used.)

We restrict our considerations to $X$, a smooth projective variety of dimension $d$ over $k$. Let $\mathscr{L}$ be a very ample $G$-invariant line bundle on $\bar{X}$, and let $\bar{\mu}: \bar{X} \rightarrow \mathbb{P}^{n-1}(\bar{k})$ be the corresponding embedding. We define $e(\mathscr{L})$ to be the degree of the image of $\bar{\mu}(\bar{X})$ as a subvariety of $\mathbb{P}^{N-1}(\bar{k})$. Following [3, $\S 1.7]$, we define the degree as follows. The function $f(n)=\operatorname{dim}_{\bar{k}}\left(\Gamma\left(\mathscr{L}^{n}\right)\right)$ is a polynomial function of degree $d$ for sufficiently large $n$ : so we define $e(\mathscr{L})$, the degree of $\mathscr{L}$, by saying that

$$
f(n)=\frac{e(\mathscr{L})}{d !} n^{d}+\text { lower terms }
$$

for sufficiently large $n$. Since $f(n)$ takes integer values, $e(\mathscr{L})$ is an integer.

We define the Pic-period of $X, p(X)$, to be the highest common factor of the degrees of all $G$-invariant very ample line bundles on $\bar{X}$. For curves, this reduces to the normal definition of the period.

Theorem 1.1. Let $X$ be a normal projective variety over $k$ of dimension $d$ with function field $K$. Let $D$ be a central division algebra of index $n$ over $K$ whose class in the Brauer group lies in the image of $\operatorname{Br}(k)$. Let $p(X)$ be the Pic-period of $X$, and let $q$ be the largest factor of $p(X)$ whose prime factors all divide 
$n$. Then there is a central division algebra $E$ over $k$ such that $D$ is similar to $E \otimes_{k} K$ and the index of $E$ divides $n q$. In particular, if $(n, p(X))=1$, $D \simeq E \otimes_{k} K$ for suitable $E$.

Proof. It is enough to prove this when $D$ has index a power of a prime $p$. Consider a sheaf of maximal orders $\mathscr{O}_{A}$ on $X$ whose stalk at the generic point of $X$ is $D$. Since the Brauer class of $D$ lies in the image of $\operatorname{Br}(k)$, and the stalk at a point of codimension 1 is unique up to conjugacy in $D$, such a stalk must be an Azumaya algebra; therefore, there is an open subset $U$ of $X$, containing all points of codimension 1 such that $\mathscr{O}_{A} \mid U$ is a sheaf of Azumaya algebras. By shrinking $U$ if necessary, we may assume that $U$ is smooth. Let $j: Y \rightarrow U$ be the associated Brauer-Severi scheme over $U .\left(\overline{\mathscr{O}}_{A} \mid \bar{U}\right)$ is a split Azumaya algebra: so $\left(\overline{\mathscr{O}}_{A} \mid \bar{U}\right) \simeq \operatorname{End}(\mathscr{E})$ for some choice of vector bundle $\mathscr{E}$ over $\bar{U}$; we may replace $\mathscr{E}$ by $\mathscr{E} \otimes j^{*} \mathscr{L}$ if we wish where $\mathscr{L}$ is any line bundle over $\bar{U} . \bar{j}: \bar{Y} \rightarrow \bar{U}$ becomes the projective space bundle $\mathbb{P}_{\bar{U}}(\mathscr{E}) \rightarrow \bar{U}$. Associated to this is the universal quotient bundle $\mathscr{O}(1)$ on $\mathbb{P}_{\bar{U}}(\mathscr{E})$. By [3, III Example 12.5], $\operatorname{Pic}(\bar{Y}) \simeq \mathbb{Z} \oplus \operatorname{Pic}(\bar{U})$; every line bundle is of the form $\mathscr{O}(1) \otimes{ }^{n} \otimes j^{*} \mathscr{L}$ for $n \in \mathbb{Z}, \mathscr{L}$ a line bundle on $\bar{U}$. Moreover, $\operatorname{Pic}(\bar{U}) \simeq \operatorname{Pic}(\bar{X})$ since $U$ contains all points of codimension 1 in $X$.

Let $i: \operatorname{spec} K \rightarrow U$ be the generic point of $U$. We form the pullback

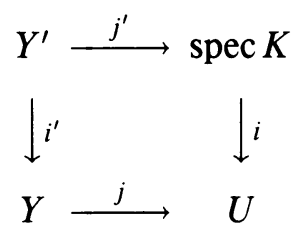

$Y^{\prime}$ is the Brauer-Severi variety of $D$ over $K$. We form the commutative diagram with exact rows and columns:

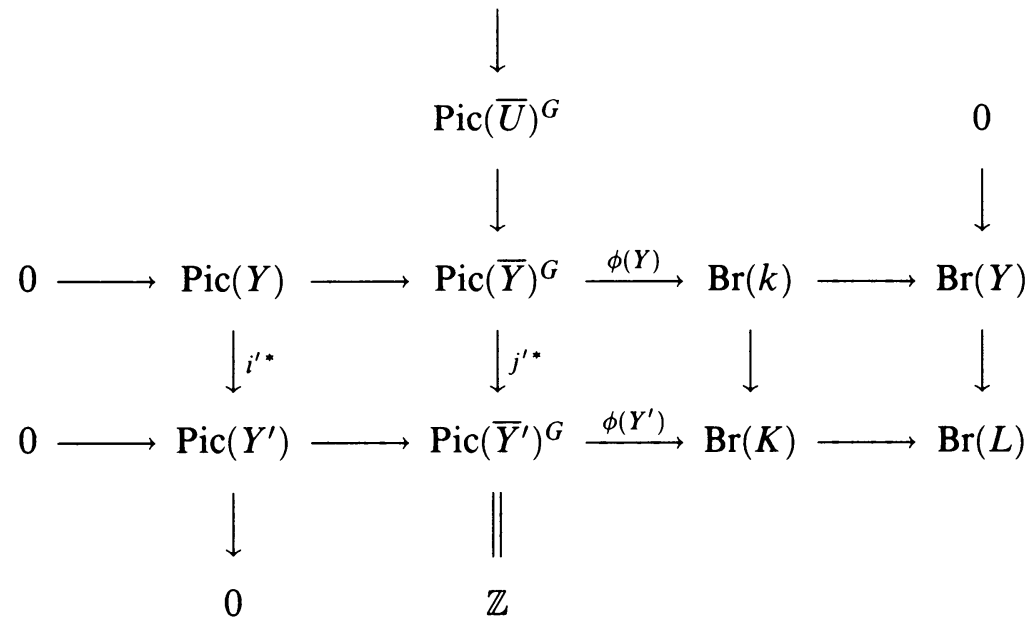

Here $L$ is the function field of $Y$ (and hence of $Y^{\prime}$ ).

The rows are exact since they arise from the Hochschild-Serre spectral sequence. The first column is exact, because $Y$ is smooth and $Y^{\prime}$ is a localization of it. The second column is exact because it arises from applying $G$-invariants 
to a short exact sequence. The rightmost vertical map is an injection because $Y$ is smooth. Consider $\phi\left(Y^{\prime}\right)\left(j^{\prime *}(\mathscr{O}(1))\right)=-[D]$. By assumption, there is a central division algebra $E$ over $k$ such that $\left[E \otimes_{k} K\right]=[D]$. Now a standard diagram chase shows that there is some element of $\operatorname{Pic}(\bar{Y})^{G}$ that maps onto $j^{\prime *}(\mathscr{O}(1))$ under $j^{\prime *}$. We deduce that it was possible to choose $\mathscr{E}$ to be $G$-invariant and so $\mathscr{O}(1)$ is also.

We fix such a $G$-invariant $\mathscr{E}$; then $\phi(Y)(\mathscr{O}(1))=-[E]$; where $\left[E \otimes_{k} K\right]=$ [D]. If $\mathscr{L}$ is a $G$-invariant line bundle on $\bar{U}$, then $\phi(Y)\left(\mathscr{O}(1) \otimes j^{*} \mathscr{L}\right)=$ $-[E]+\phi(U)(\mathscr{L})$ and since $\phi(U)(\mathscr{L})$ is split on $U$, and hence split by $K$, we may also look at these line bundles to find division algebras $D^{\prime}$ such that $D^{\prime} \otimes_{k} K$ is similar to $D$.

We wish to consider the global sections of such a line bundle on $\bar{Y}$, but $\left.\Gamma\left(\bar{Y}, \mathscr{O}(1) \otimes j^{*} \mathscr{L}\right) \equiv \Gamma\left(\bar{U}, j_{*}(\mathscr{O}(1)) \otimes \mathscr{L}\right)\right)=\Gamma(\bar{U}, \mathscr{E} \otimes \mathscr{L})=\Gamma\left(\bar{X}, \mu_{*}(\mathscr{E} \otimes \mathscr{L})\right)$ where $\mu: \bar{U} \rightarrow \bar{X}$ is the inclusion. This equals $\Gamma\left(\bar{X}, \mu_{*}(\mathscr{E}) \otimes \mathscr{L}\right)$ where we abuse the isomorphism of $\operatorname{Pic}(\bar{U})$ and $\operatorname{Pic}(\bar{X})$.

Lemma 1.2 (see after the end of this theorem) shows that $\mu_{*}(\mathscr{E})$ is a coherent sheaf on $\bar{X}$. It has rank $r=p^{t}$, the index of $D$ by construction. Let $\mathscr{L}$ be a very ample $G$-invariant line bundle such that the maximal power of $p, p^{s}$, that divides the Pic-period of $X$ is the maximal power of $p$ dividing the degree of $\mathscr{L}$.

Following [3, §1.7], we see that for sufficiently large $m$,

$$
f(m)=\operatorname{dim}\left(\Gamma\left(\bar{X}, \mu_{*}(\mathscr{E}) \otimes \mathscr{L}^{m}\right)\right)
$$

is a polynomial function of $m$ of degree $d$; moreover,

$$
f(m)=\frac{p^{t} e(\mathscr{L})}{d !} m^{d}+\text { lower terms }
$$

for sufficiently large $m$, where $e(\mathscr{L})$ is the degree of $\mathscr{L}$. Let us assume that we have chosen $m$ to be sufficiently large: then

$$
p^{t} e(\mathscr{L})=\sum_{j=0}^{d}(-1)^{j}\left(\begin{array}{l}
d \\
j
\end{array}\right) \operatorname{dim} \Gamma\left(\bar{X}, \mu_{*}\left(\mathscr{E} \otimes \mathscr{L}^{m+d-j}\right)\right) .
$$

Hence there is an integer $n$ such that the exponent of the highest power of $p$ dividing $\operatorname{dim} \Gamma\left(\bar{X}, \mu_{*}(\mathscr{E}) \otimes \mathscr{L}^{n}\right)$ is at most $s+t$. For this choice of $n$, the highest power of $p$ dividing the index of $\phi(Y)\left(\mathscr{O}(1) \otimes j^{*} \mathscr{L}^{n}\right)$ is a factor of $p^{s+t}$. The $p$-primary part of the division algebra $E^{\prime}$, such that $E^{\prime \circ}$ represents this Brauer class, then fulfills the requirements.

In the course of the proof of Theorem 1.1, we referred to Lemma 1.2 which remains to be proved. We prove this next.

Lemma 1.2. Let $X$ be a normal variety and let $\mu: U \rightarrow X$ be an open subvariety containing all points of codimension 1 . Let $\mathscr{E}$ be a vector bundle on $U$; then $\mu_{*}(\mathscr{E})$ is a coherent sheaf of reflexive modules on $X$.

Proof. It is standard [3, II, Exercise 5.15] that there is a coherent sheaf $S$ on $X$ such that $S \mid U=\mathscr{E}$. We may ensure that $S$ is reflexive by replacing it by $S^{* *} \simeq \operatorname{Hom}\left(\operatorname{Hom}\left(\mathscr{S}, \mathscr{O}_{X}\right)\right) ;$ since $S \mid U \simeq \mathscr{E}$, a vector bundle, $S^{* *} \mid U \simeq \mathscr{E}$ also. $S^{* *}$ is reflexive. But, if $V$ is an open affine, then $\Gamma\left(V, S^{* *}\right)=\bigcap_{p \in V^{(1)}} S_{p}^{* *}$ where the intersection ranges over all points of codimension 1 in $V$; but since 
$U$ contains all points of codimension 1, this is $\bigcap_{p \in V^{(1)} \cap U} \mathscr{E}_{p}=\Gamma(V \cap U, \mathscr{E})$. Therefore, $S^{* *} \simeq \mu_{*}(\mathscr{E})$.

We deduce our main theorem from Theorem 1.

Theorem 1.3. Let $D, E$ be central division algebras over $k$ of indices $m$ and $n$ respectively. Let $\mathrm{Br}-S(E)$ be the Brauer Severi variety of $E$, and let $K$ be its function field. Then the index of $D \otimes_{k} K$ is the minimum of the indices of the central simple algebras $\left\{D \otimes E^{*}, i=1\right.$ to $\left.n\right\}$.

Proof. $\mathrm{Br}-S(E) \times_{k} \bar{k} \simeq \mathbb{P}^{n-1}(\bar{k}) . \mathscr{O}(1)$ is a $G$-invariant line bundle of degree 1. Therefore, if $D^{\prime}$ is the central division algebra in the Brauer class of $D \otimes_{k} K$, there is a division algebra $C$ over $k$ such that $D^{\prime} \simeq C \otimes_{k} K$ by Theorem 1 . Since $D^{0} \otimes_{k} C$ is split by $K$, and the only part of the Brauer group split by $K$ is the subgroup generated by $E, C$ is similar to $D \otimes E^{i}$ for some $i$.

This shows that the index of $D \otimes_{k} K$ is the index of some $D \otimes E^{i}$. On the other hand, for arbitrary $i$, the index of $D \otimes_{k} K$ is the same as the index $\left(D \otimes E^{i}\right) \otimes_{k} K$, which is not higher than the index of $D \otimes E^{i}$.

There is another proof of Theorem 1.3 from a different point of view. If $S$ is a central simple algebra, $K_{0}(S) \simeq \mathbb{Z}$; however, the free module of rank 1 defines a distinguished class, and it is more natural to write $K_{0}(S) \simeq \frac{1}{n} \mathbb{Z}$ where $S \simeq M_{n}(D)$ for some division algebra $D$. Thus, $K_{0}$ allows us to determine the index of a central simple algebra.

If $D$ and $E$ are central division algebras over $k$, we wish to determine $K_{0}\left(D \otimes_{k} K\right)$ where $K$ is the function field of $\mathrm{Br}-S(E)$. Quillen has computed the $K$-theory of $\mathrm{Br}-S(E)$, more accurately, the $K$-theory of coherent sheaves on $\mathrm{Br}-S(E)$, which allows us to compute the $K$-theory of $D \otimes \mathrm{Br}-S(E)$, by which we mean the category of coherent sheaves on $\operatorname{Br}-S(E)$ with a right $D \otimes_{k} \mathscr{O}(\mathrm{Br}-S(E))$ structure. It is a simple matter to prove that the map from $K_{0}(D \otimes \mathrm{Br}-S(E)) \rightarrow K_{0}(D \otimes K)$ is surjective, and this gives a simple calculation of $K_{0}(D \otimes K)$. Below we shall fill out this sketch.

Given an irreducible nonsingular variety $X$, we define $K_{*}(D \otimes X)$ to be the Quillen $K$-theory of the abelian category $\operatorname{Coh}(D \otimes X)$ of sheaves of modules for the sheaf of algebras $D \otimes \mathscr{O}(X)$, coherent as $\mathscr{O}(X)$ sheaves.

Lemma 1.4. The natural map from $K_{0}(D \otimes X)$ to $K_{0}(D \otimes K(X))$ is surjective where $K(X)$ is the function field of $X$.

Proof. Let $M$ be a module for $D \otimes K(X)$; it is standard that there is a coherent $\mathscr{O}(X)$ sheaf $\mathscr{P}$, a subsheaf of the sheaf which associates $M$ to each open affine subset of $X$, such that $\mathscr{P} \otimes K(X) \simeq M$. Since $D$ is finite-dimensional over $k$ the sheaf $\mathscr{P} D$ inside $M$ is still coherent and $\mathscr{P} D \otimes_{D \otimes \mathscr{O}(X)} K(X) \simeq M$ so the image of $[\mathscr{P} D]$ in $K_{0}(D \otimes K(X))$ is $[M]$, and the map is surjective.

It remains to calculate $K_{*}(D \otimes \mathrm{Br}-S(E))$; this is a very mild generalization of Quillen's calculation of $K_{*}(\mathrm{Br}-S(E))$, with which we shall assume that the reader is familar in the following proof. We refer the reader to $\S 8.4$ of [6] for unexplained terminology in the following.

Let $E$ be a division algebra of index $r$; Quillen constructs a bundle $J$ on $\mathrm{Br}-S(E)$ whose sheaf of endomorphism rings is $E \otimes \mathscr{O}(X)$ where $E$ acts on the right on $J$. We define $J_{i}$ to be the $i$-fold tensor power of $J$ and the sheaf of endomorphism rings of $J_{i}$ is $E^{\otimes i} \otimes \mathscr{O}(X)$. We have a functor from left 
$E^{\otimes i}$ modules to sheaves on $\mathrm{Br}-S(E)$ by $J_{i} \otimes_{E^{\otimes i}}$ which restricts to a functor from left $E^{\otimes i}$-, right $D$ bimodules, that is right $\left(D \otimes\left(E^{0}\right)^{\otimes i}\right)$ modules, to $\operatorname{Coh}(D \otimes \mathrm{Br}-S(E))$. Using this, we state the theorem

Theorem 1.5. We have isomorphisms

$$
\bigoplus_{i=0}^{r-1} K_{i}\left(D \otimes\left(E^{0}\right) \otimes i\right) \rightarrow K_{i}(D \otimes X)
$$

by $\left(x_{n}\right) \rightarrow \sum_{i=1}^{r-1}\left(J_{i} \otimes_{E^{\otimes i}}-\right)_{*}\left(x_{n}\right)$.

Proof. Recall that a sheaf $\mathscr{P}$ on $\mathrm{Br}-S(E)$ is said to be regular if

$$
H^{i}(\mathscr{P} \otimes \bar{k}(-i))=0 \quad \text { for } i>0 .
$$

On the subcategory of regular sheaves, there are functors $T_{i}$ to left modules for $E^{\otimes i}$ such that there is an exact sequence

$$
0 \rightarrow J_{r-1} \otimes_{E^{r-1}} T_{r-1}(F) \rightarrow \cdots \rightarrow \mathscr{P}(X) \otimes_{k} T_{0}(F) \rightarrow F \rightarrow 0 .
$$

On the subcategory of $\operatorname{Coh}(D \otimes X)$ of regular sheaves, $T_{i}$ is a functor to left $E^{\otimes i}$ right $D$ bimodules, since the functoriality of $T_{i}$ implies there is a right $D$ action on $T_{i}(F)$.

Finally, given any sheaf $\zeta$ on $\mathrm{Br}-S(E), \zeta \otimes\left(\left(\Lambda^{r} J\right)^{\vee}\right)^{n}$ is regular for sufficiently large $n$. This, together with the sequence

$$
0 \rightarrow \Lambda^{r} J \rightarrow \Lambda^{r-1} J \rightarrow \cdots \rightarrow J \rightarrow \mathscr{O} \rightarrow 0
$$

allows us to prove that the $K$-theory of $\operatorname{Coh}(D \otimes X)$ is naturally isomorphic to the $K$-theory of the regular sheaves in $\operatorname{Coh}(D \otimes X)$, and the functors $T_{i}$ show that the $K$-theory of regular sheaves in $\operatorname{Coh}(D \otimes X)$ is as required by the arguments of $\S \S 8.4$ and 8.2 of [6].

For the statement of the next consequence, which is equivalent to Theorem 1.3 , we identify, for a central simple algebra $S, K_{0}(S)$ with $\frac{1}{n} \mathbb{Z}$, where $n$ is chosen in such a way that the class of the free $S$-module of rank 1 maps to the elements $1 \in \frac{1}{n} \mathbb{Z}$.

Theorem 1.6. $K_{0}(D \otimes K(\mathrm{Br}-S(E)))=\sum r^{i} K_{0}\left(D \otimes\left(E^{0}\right)^{\otimes i}\right)$. Therefore, the index of $D \otimes K(\mathrm{Br}-S(E))$ is the highest common factor of the indices of $D \otimes\left(E^{0}\right)^{i}$ as $i$ varies.

Proof. Since the rank of $J_{i}$ is $r^{i}$, the combined map

$$
J_{i} \otimes_{E^{\otimes i}}-: K_{0}\left(D \otimes\left(E^{0}\right)^{\otimes i}\right) \rightarrow K_{0}(D \otimes \mathrm{Br}-S(E)) \rightarrow K_{0}(D \otimes K(\mathrm{Br}-S(E))
$$

is multiplication by $r^{i}$. The last sentence is a simple calculation.

In Theorem 1.3., we stated that the index of $D \otimes K(\mathrm{Br}-S(E))$ was the minimum of the indices of $D \otimes E^{i}$ as $i$ varies. The reader may check by splitting $E$ as a tensor product of division algebra of prime power index that the theorem above is actually equivalent to 1.3 .

\section{Applications}

Theorem 1.3 has a number of consequences which we shall outline in this section. These fall into two types: the calculation of the index of a division algebra under certain generic central extensions; and the construction of division algebras with certain special properties. We begin with the first type, and we note a simple consequence. 
Theorem 2.1. Let $D$ be a division algebra of index $n$ with center $k$. Let $n=s t$ where every prime dividing $n$ divides $t$, and $s$ and $t$ are integers. Let $K$ be the function field of the Brauer-Severi variety of $D^{t}$. The $D \otimes_{k} K$ has index $n$. Proof. The class of $D \otimes\left(D^{t}\right)^{i}$ is $(1+i t)[D]$ where $(1+i t)$ is coprime to $n$. $D \otimes\left(D^{t}\right)^{i}$ has index $n$ for all $i$, and so, by $1.3, D \otimes K$ has index $n$.

This has the following consequence pointed out by Saltman. We recall that the generic division algebra of index $n$ and exponent $t$ over $k$ is the division algebra $E \otimes_{L} K$ where $E$ is a generic division algebra over $k$ with center $L$ and $K$ is the generic splitting field of $E^{t}$. A division algebra is indecomposable if it is not a tensor product of smaller index division algebras.

Theorem 2.2. Let $D$ be a division algebra of index $p^{a}$ with center $k$ such that $D^{p}$ has index $p^{a-1}$. Let $t \geq 2$, and let $K$ be the generic splitting field of $D^{p^{t}}$. Then $D \otimes K$ has index $p^{a}, D^{p} \otimes K$ has index $p^{a-1}$, and so, $D \otimes K$ is indecomposable.

In particular, the generic division algebra of index $p^{a}$ and exponent $p^{t}$ is indecomposable.

Proof. The previous theorem shows that the indices of $D \otimes K$ and $D^{p} \otimes K$ are as stated. If $D \otimes K \simeq E \otimes_{k} F$, then $D^{p} \otimes K \simeq E^{p} \otimes F^{p}$ and its index must be less than $p^{a-1}$.

We shall extend this later to give examples of a division algebra $D$ of prime power index such that the sequence of indices of $D, D^{p}, D^{p^{2}}, \ldots$ is any given strictly decreasing sequence.

Next, we extend 1.3 to the function field of a product of Brauer-Severi varieties. If $K$ is the function field of $\prod_{i} \mathrm{Br}-S\left(E_{i}\right)$, for central division algebras $\left\{E_{i}\right\}$ over $k$, then the subgroup of the Brauer group in the kernel of $\operatorname{Br}(k) \rightarrow \operatorname{Br}(K)$ is generated by $\left\{\left[E_{i}\right]\right\}$.

Theorem 2.3. Let $K$ be the function field of $\prod_{i} \mathrm{Br}-S\left(E_{i}\right)$ where each $E_{i}$ is a central division algebra over $k$. Then the index of $D \otimes_{k} K$ where $D$ is a central division algebra over $k$ is the minimum of the indices of $D \otimes E_{1}^{i_{1}} \otimes \cdots \otimes E_{n}^{i_{n}}$.

Proof. Let $K_{1}$ be the function field of $\prod_{i \neq 1} \mathrm{Br}-S\left(E_{i}\right)$. Then $K$ is the function field of $\mathrm{Br}-S\left(E_{1} \otimes_{k} K_{1}\right)$. By Theorem 1.3, the index of $\left(D \otimes_{k} K_{1}\right) \otimes_{K_{1}} K=D \otimes K$ is the minimum of the indices of $\left(D \otimes E_{1}^{j}\right) \otimes K_{1}$ as $j$ varies.

By induction, the index of $\left(D \otimes_{k} E_{1}^{j}\right) \otimes K_{1}$ is the minimum of the indices of $D \otimes_{k} E_{1}^{j} \otimes E_{2}^{j_{2}} \otimes \cdots \otimes E_{n}^{j_{n}}$ so the result follows.

We consider the following general situation. We define central division algebras $D$ and $E$ over $k$ to be independent if there are commutative fields $K^{\prime}$ and $K^{\prime \prime}$ such that $D \otimes_{k} K^{\prime}$ is split and, for any $i$, the index of $E^{i} \otimes_{k} K^{\prime}$ is the index of $E^{i}$ whilst $E \otimes_{k} K^{\prime \prime}$ is split and, for any $i$, the index of $D^{i} \otimes_{k} K^{\prime \prime}$ is the index of $D^{i}$.

Theorem 2.4. Let $D, E$ be independent central division algebras over $k$. Let $K$ be the function field of the Brauer-Severi variety of $D \otimes E^{0}$. Then the index of $D^{i} \otimes_{k} K$ is the highest common factor of the indices of $D^{i}$ and $E^{i}$.

Proof. Fix $i$. The index of $D^{i} \otimes_{k} K$ is the minimum of the indices of $D^{i} \otimes$ $\left(D \otimes E^{0}\right)^{j}=D^{i+j} \otimes\left(E^{0}\right)^{j}$ by Theorem 1.3. Let $p$ be a prime number dividing $l=$ h.c.f. $\left\{\operatorname{ind}\left(D^{i}\right)\right.$, ind $\left.\left(E^{i}\right)\right\}$ and let $p^{s}$ be the highest power of $p$ dividing 
$l$. For fixed $j$, let $p^{t}$ be the highest power of $p$ dividing $(i+j)$ and $j$; then $p^{t}$ divides $i$. Either $p^{t+1} \nmid(i+j)$ or $p^{t+1} \nmid j$. In the first case, let $K^{\prime \prime}$ be a field that splits $E$ such that the index of $D^{h} \otimes_{k} K^{\prime \prime}$ equals the index of $D^{h}$ for all $h$. Then, the index of $D^{i+j} \otimes_{k}\left(E^{0}\right)^{j}$ is a multiple of the index of $D^{i+j} \otimes_{k}\left(E^{0}\right)^{j} \otimes_{k} K^{\prime \prime}$ which is the index of $D^{i+j} \otimes_{k} K^{\prime \prime}$ which equals the index of $D^{i+j}$. Since $p^{t+1} \nmid(i+j)$ and $p^{t} \mid i$, the $p$-component of $D^{i}$ is a power of the $p$-component of $D^{i+j}$ and so the $p$-primary part of the index of $D^{i}$ divides the index of $D^{i+j}$ which divides the index of $D^{i+j} \otimes\left(E^{0}\right)^{j}$ by the above. So, $p^{s}$ divides the index of $D^{i+j} \otimes\left(E^{0}\right)^{j}$. Similarly, if $p^{t+1} \nmid j$, we tensor with $K^{\prime}$ where $K^{\prime}$ splits $D$ and leaves the index of $E^{h}$ alone and follow the same argument. We conclude in either case that $p^{s}$ divides the index of $D^{i} \otimes_{k} K$ where $p^{s}$ is the highest power of $p$ dividing $l$. So, $l$ divides the index of $D^{i} \otimes_{k} K$.

The converse is clear since $D^{i} \otimes_{k} K$ is similar to $E^{i} \otimes_{k} K$.

Let $S$ be a central simple algebra over $k$ of dimension $n^{2}$ over $k$ and index $s$; so $n=s t$ and $s \simeq M_{t}(D)$ where $D$ is a division algebra. Let $\sigma \in H^{1}\left(\bar{k} / k, P G l_{n}(\bar{k})\right)$ be the cohomology class corresponding to $S$. Let $q$ be an integer dividing $n, n=q r$, and let $K\left(\operatorname{Gr}\left(\begin{array}{l}n \\ r\end{array}\right)(\sigma)\right)$ be the function field of the twisted form, $\operatorname{Gr}\left(\begin{array}{l}n \\ r\end{array}\right)(\sigma)$, corresponding to $\sigma$, of the Grassmannian, $\operatorname{Gr}\left(\begin{array}{l}n \\ r\end{array}\right)$, of $r$-dimensional subspaces of $n$-dimensional space. In [7], we considered this field since $S \otimes_{k} K\left(\operatorname{Gr}\left(\begin{array}{l}n \\ r\end{array}\right)(\sigma)\right) \simeq M_{q}\left(S^{\prime}\right)$ for a simple algebra $S^{\prime}$, and is universal with this property since $\operatorname{Gr}\left(\begin{array}{l}n \\ r\end{array}\right)(\sigma)$ has a point in a field $L$ if and only if $S \otimes$ $L \simeq M_{q}\left(S_{1}\right)$. This variety and its function field have also been investigated by A. Blanchet in [2]. We wish to determine the index of $S \otimes_{k} K\left(\operatorname{Gr}\left(\begin{array}{l}n \\ r\end{array}\right)(\sigma)\right)$. It clearly divides h.c.f. $\{r, s\}$. We shall prove it is equal to this by finding a suitable field $L$ such that $S \otimes_{k} L \simeq M_{q}\left(S_{1}\right)$ for some algebra $S_{1}$, of index h.c.f. $\{r, s\}$. Then the universal property of $\operatorname{Gr}\left(\begin{array}{l}n \\ r\end{array}\right)(\sigma)$ allows us to pull this back to $S \otimes_{k} K\left(\operatorname{Gr}\left(\begin{array}{l}n \\ r\end{array}\right)(\sigma)\right)$.

Theorem 2.5. Let $S$ be a central simple algebra of dimension $n^{2}$ over $k$ and of index $s$ where $n=s t$. Let $n=q r$ for integers $q$ and $r$ and let $K$ be the function field of $\operatorname{Gr}\left(\begin{array}{l}n \\ r\end{array}\right)(\sigma)$ where $\operatorname{Gr}\left(\begin{array}{l}n \\ r\end{array}\right)(\sigma)$ is the twisted form of $\operatorname{Gr}\left(\begin{array}{l}n \\ r\end{array}\right)$, the Grassmannian, corresponding to the cocycle $\sigma \in H^{1}\left(\bar{k} / k, P G L_{N}(\bar{k})\right)$ determined by $S$. Then the index of $S \otimes_{k} K$ is h.c.f. $\{r, s\}$.

Proof. Let $D$ be a generic division algebra of index $R$ with center $C$, and let $L$ be the generic splitting field of $\left(S^{\circ} \otimes_{k} C\right) \otimes_{C} D$. Then $S \otimes_{k} L=M_{q}\left(S_{1}\right)$ for some central simple algebra, and as pointed out in the discussion just before the present theorem, it suffices to show that the index of $S_{1}$ is equal to h.c.f. $\{r, s\}$ i.e. equal to the h.c.f. of the indices of $S$ and $D$.

It is easy to see that $S_{1}=D \otimes_{C} L$. If we show that $S \otimes_{k} C$ and $D$ are independent then the index of $S_{1}$ is the h.c.f. of the indices of $S \otimes_{k} C$ and $D$. Furthermore, since $C$ is unirational over $k$, the index of $S^{i}$ is the index of $S^{i} \otimes C$. Taking $i=1$ then proves the theorem.

We now proceed by showing that $S \otimes_{k} C$ and $D$ are independent. $D$ has a splitting field $K^{\prime}$, rational over $k$. The index of $\left(S \otimes_{k} C\right)^{i} \otimes_{C} K^{\prime}$ is the index of $S^{i}$ which is, as pointed out above, equal to the index of $S^{i} \otimes_{k} C$.

Let $l \supset k$ be an algebraic splitting field of $S$ and let $K^{\prime \prime}=l \otimes_{k} C, l \otimes_{k} D$ is a generic division algebra over $K^{\prime \prime}$, so the index of $K^{\prime \prime} \otimes_{C} D^{i}=l \otimes_{k} D^{i}$ equals 
the index of $D^{i}$. This shows that $S \otimes_{k} C$ and $D$ are independent.

Finally, we can fill in a hole in the paper [7]. We showed in that paper that if $D$ is a central division algebra of index and exponent $n$ and $l \supset k$ is a separable extension of dimension dividing $n$, then there is a regular field extension $K \supset k$ such that $D \otimes_{k} K$ contains $l \otimes_{k} K$ and remains a division algebra. In the next part, we use the notation of this paper freely since the question is of interest only if one has read this paper.

Let us suppose that $[l: k]=q$ where $n=q r$. Let $K$ be the function field of the variety $V=\operatorname{Gr}\left(\begin{array}{l}n \\ r\end{array}\right)(\sigma) \uparrow_{k}^{l} \downarrow_{k}^{l}$ (we refer the reader to [7] for the notation and description of this variety). Then $V$ has a point in the field $F$ if and only if $D \otimes_{k} F$ contains $l \otimes_{k} F$ (at least when $l \otimes_{k} F$ is a field; more care is required in general). We wish to prove that $D \otimes_{k} K$ is a division algebra even when the exponent of $D$ is less than $n$. We shall prove this by finding a field $F$ such that $D \otimes_{k} F \supset l \otimes_{k} F$ and is a division algebra from which the result follows at once since $D \otimes_{k} K$ specializes to $D \otimes_{k} F$.

Theorem 2.6. Let $D$ be a central division algebra over $k$ of index $n$. Let $l \supset k$ be a separable extension of dimension $q$ where $n=q r$ for $r$ an integer. Let $K$ be the function field of $\operatorname{Gr}\left(\begin{array}{l}n \\ r\end{array}\right)(\sigma) \uparrow_{k}^{l} \downarrow_{k}^{l}$. Then $D \otimes_{k} K$ is a division algebra and $l \otimes_{k} K$ embeds in it.

Proof. For the notation see [7]. We construct a field $F$ such that $D \otimes_{k} F$ is a division algebra and $l \otimes_{k} F$ embeds in it.

Let $E$ be a generic division algebra of index $n$ over $k$ with center $C$. Let $C^{\prime \prime} \supset C$ be the function field of

$$
\operatorname{Gr}\left(\begin{array}{l}
n \\
r
\end{array}\right)(\tau) \uparrow_{C}^{C \otimes l} \downarrow_{C}^{C \otimes l}
$$

where $\tau$ is the class in $H^{1}\left(\bar{C} / C, P G l_{n}(\bar{C})\right)$ determined by $E$. Then, by Theorem 3.9 of [7], $C^{\prime \prime} \otimes_{C} E$ is a division algebra of index and exponent $n$ in which $C^{\prime \prime} \otimes_{k} l$ embeds. We call this construction the universal division algebra over $k$ of index $n$ containing $l$. If $K_{1} \supset k$ is any field such that $K_{1} \otimes_{k} l$ is a field, then the simple algebra of quotients of $K_{1} \otimes_{k}\left(C^{\prime \prime} \otimes_{C} E\right)$ is the universal division algebra over $K_{1}$ of index $n$ containing $K_{1} \otimes_{k} l$.

We shall prove that $C^{\prime \prime} \otimes_{C} E$ and $C^{\prime \prime} \otimes_{k} D$ are independent in the sense of Theorem 2.4. Then, by taking $F$ to be Brauer-Severi variety of $D^{0} \otimes_{k}\left(E \otimes_{C} C^{\prime \prime}\right)$ our result follows from 2.4 .

First of all, if we take $K_{1}$ to be the generic splitting field of $D$ over $k$, $K_{1} \otimes_{k} l$ is a field, so the simple algebra of fractions of $K_{1} \otimes_{k}\left(E \otimes_{C} C^{\prime \prime}\right)$ is a division algebra of index and exponent $n$ by the preceding remarks. So, set $K^{\prime}$ to be the field of fractions $K_{1} \otimes_{k} C^{\prime \prime}$.

On the other hand, $E$ has a splitting field $C^{\prime} \supset C \supset k$ rational over $k$, and the variety $\left(\operatorname{Gr}\left(\begin{array}{l}n \\ r\end{array}\right)(\sigma) \uparrow_{C}^{C \otimes l} \downarrow_{C}^{C \otimes l}\right) \otimes_{C} C^{\prime}$ becomes simply $\operatorname{Gr}\left(\begin{array}{l}n \\ r\end{array}\right) \uparrow_{C^{\prime}}^{C^{\prime} \otimes l} \downarrow_{C^{\prime}}^{C^{\prime} \otimes l}$ which is rational over $C^{\prime}$ and hence its function field $K^{\prime \prime}$ is rational over $k$. Therefore $\left(E \otimes_{C} C^{\prime \prime}\right) \otimes_{C^{\prime \prime}} K^{\prime \prime}$ is split but $\left(D^{i} \otimes_{k} C^{\prime \prime}\right) \otimes_{C^{\prime \prime}} K^{\prime \prime}$ has the same index as $D^{i}$. Thus we have shown that $E \otimes_{C} C^{\prime \prime}$ and $D \otimes_{k} C^{\prime \prime}$ are independent and our result follows.

It follows at once that given any division algebra $D$ of index $n$ over $k$, there exists a regular field extension $K \supset k$ such that $D \otimes_{k} K$ is a cyclic division algebra. In order to prove this, one notes that there exists some unirational field 
$k^{\prime} \supset k$ which has a field extension $L \supset k^{\prime}$ which is cyclic of dimension $n$. If $K \supset k^{\prime}$ is the generic field such that $\left(D \otimes_{k} k^{\prime}\right) \otimes_{k^{\prime}} K$ contains $L \otimes_{k^{\prime}} K$, then $D \otimes_{k} K$ is a cyclic division algebra by Theorem 2.6. Of course, we could have found some field $K(G)$ such that $D \otimes K(G)$ is a crossed product division algebra with Galois group $G$ for any group $G$ of order $n$.

Now we give some constructions of division algebras. First of all, we construct a division algebra, $D$, of index $p^{n}$ such that the sequence of integers, index $(D)$, index $\left(D^{p}\right)$, index $\left(D^{p^{2}}\right), \ldots$ is any given strictly decreasing sequence of powers of $p$. For this we need the following result.

Theorem 2.7. Let $D$ be a central division algebra over $k$ of index $p^{a}$. Assume that $D^{p^{b}}$ is a division algebra of index and exponent $p^{c}$. Let $d<c$. Let $E$ be a generic division algebra of index $p^{d}$ over $k$ with center $C$. Let $K \supset C$ be the function field of the Brauer-Severi variety of $D^{p^{b}} \otimes E^{0}$. Then $D \otimes_{k} K$ is a division algebra, $D^{p^{i}} \otimes K$ has the same index as $D^{p^{i}}$ for $i<b$, and for $i \geq b$, $D^{p^{i}} \otimes K$ has index and exponent $\max \left(p^{d-i+b}, 1\right)$.

Proof. For $i \geq b$, we may apply Theorem 2.4 since $D^{p^{b}} \otimes C$ and $E$ are independent. We obtain that the index of $D^{p^{i}} \otimes K$ is the h.c.f. of the indices of $D^{p^{i}}$ and $E^{p^{i-b}}$.

By hypothesis, $D^{p^{b}}$ and $E$ have exponent equal to index, so we obtain that the index of $D^{p^{i}}$ is $\max \left(p^{c-i+b}, 1\right)$ and the index of $E^{p^{i-b}}$ is $\max \left(p^{d-i+b}, 1\right)$. The result follows from this, using $d<c$.

Assume $i<b$. We have to calculate the indices of $D^{p^{i}} \otimes\left(D^{p^{b}} \otimes E^{0}\right)^{j}$ for varying $j$. Let $L \supset C$ be a splitting field of $E$ rational over $k$; then $D^{p^{i}} \otimes$ $\left(D^{p^{b}} \otimes E^{0}\right)^{j} \otimes_{C} L$ is similar to $D^{p^{i}+j p^{b}} \otimes_{k} L$ which has the same index as $D^{p^{i}}$ since $L \supset k$ is rational. It follows that $D^{p^{i}} \otimes_{k} K$ has the same index as $D^{p^{i}}$, as required.

Construction 2.8. We wish to construct a division algebra $D$ of index $p^{n}$ such that the sequence of numbers index $(D)$, index $\left(D^{p}\right)$, index $\left(D^{p^{2}}\right), \ldots$ is any given sequence of strictly decreasing powers of $p$. We simply apply 2.7 inductively. We begin by taking $D_{0}$ to be a generic division algebra of index $p^{n}$. We assume that $D_{i}$ is a division algebra such that the sequence $\operatorname{index}\left(D_{i}\right), \ldots, \operatorname{index}\left(D_{i}^{p^{i}}\right)$ is as required, but $\operatorname{index}\left(D_{i}^{p^{i+j}}\right)=\operatorname{index}\left(D_{i}^{p^{i}}\right) / p^{j}$. Then we apply 2.7 to pass to $D_{i+1}=D_{i} \otimes K_{i}$ for some field $K_{i}$ such that $D_{i+1}$ has the correct inductive property.

Constructon 2.9. In [8], Tognol and Wadsworth give examples of division algebras $D$ and $E$ of any odd index such that $D \otimes E^{0}$ has zero-divisors, but, as Saltman remarked, $D \otimes E$ has not, so they have no field in common.

Given any odd prime $p$, we construct a pair of division algebras $D, E$ of index $p$ such that $D \otimes E^{i}$, for $i=2 \rightarrow p-1$, has zero-divisors but $D \otimes E$ does not. We would like to thank $L$. Rowen for suggesting this. Our construction is simply to take $D_{1}$ and $E_{1}$ to be a pair of generic division algebras of index $p$ on independent sets of variables and then generically make $D_{1} \otimes E_{1}^{i}$ of index $p$ for $i=2$ to $p-1$. For this universal example, $D \otimes E$ has index $p^{2}$. In order to do this, let $D_{1}, E_{1}, F_{2}, \ldots, F_{p-1}$ be $p$ generic division algebras of index $p$ on distinct sets of indeterminates. Let $C$ be the field of fractions of the tensor 
product over $k$ of their centers. We shall abusively write $D_{1}, E_{1}, F_{2}, \ldots, F_{p-1}$ in place $D_{1} \otimes C, E_{1} \otimes C$, etc. where the tensor product in each case over the center of the division algebra. The index of $D_{1}^{i_{1}} \otimes E_{1}^{i_{2}} \otimes F_{2}^{i_{3}} \otimes \cdots \otimes F_{p-1}^{i_{p}}$ is simply the product $\operatorname{index}\left(D_{1}^{i_{1}}\right) \times \operatorname{index}\left(E_{1}^{i_{2}}\right) \times \cdots \times \operatorname{index}\left(F_{p-1}^{i_{p}}\right)$. Let $K \supset C$ be the function field of $\prod_{i=2}^{p-1} \mathrm{Br}-S\left(D_{1} \otimes E_{1}^{i} \otimes F_{i}\right)$.

By construction, the index of $D_{1} \otimes E_{1}^{i} \otimes K$ is $p$ for $i \geq 2$. By Theorem 2.3 , the index of $D_{1} \otimes E_{1} \otimes_{C} K$ is the minimum of the indices of $\left(D_{1} \otimes E_{1}\right) \otimes$ $\otimes_{i=2}^{p-1}\left(D_{1} \otimes E_{1}^{i} \otimes F_{i}\right)^{j_{i}}$. If two of the $j_{i}$ 's are not divisible by $p$, then by looking at the contribution of the algebras $F_{i}^{j_{i}} \otimes F_{i^{\prime}}^{j_{i}^{\prime}}$, we see the index is at least $p^{2}$. So this leaves checking the index of $D_{1} \otimes E_{1} \otimes\left(D_{1} \otimes E_{1}^{i} \otimes F_{i}\right)^{j_{i}}$ for some $i$ and $j_{i}$. Again this must have index at least $p^{2}$. So $D_{1} \otimes E_{1} \otimes K$ has index $p^{2}$ as required.

\section{REFERENCES}

1. M. Artin (Notes by A. Verschoren), Brauer-Severi varieties, Lecture Notes in Math., vol. 917, Springer-Verlag, 1981, pp. 194-210.

2. A. Blanchet, Function fields of generalized Brauer-Severi varieties, preprint, University of Nebraska, 1989.

3. R. Hartshorne, Algebraic geometry, Graduate Texts in Math., no. 52, Springer-Verlag, New York, 1977.

4. S. Lichtenbaum, The period-index problem for elliptic curves, Amer. J. Math. 90 (1968), 1209-1223.

5. J. S. Milne, Étale cohomology, Princeton Univ. Press, Princeton, N. J., 1980.

6. D. Quillen, Higher algebraic K-theory. I, Lecture Notes in Math., vol. 341, Springer-Verlag, 1972, pp. 85-147.

7. A. Schofield and M. Van den Bergh, Division algebra coproducts of index $n$, Trans. Amer. Math. Soc. (to appear).

8. J. Tignol and A. Wadsworth, Totally ramified valuations on finite-dimensional division algebras, Trans. Amer. Math. Soc. 302 (1987), 223-250.

School of Mathematics, University Walk, Bristol BS8 1TW, England

Department of Mathematics, Universiteitsplein 1, 2610 WilrijK, Belgium 